Archives de sciences sociales des religions

148 | octobre-décembre 2009

Bulletin Bibliographique

\title{
Bengt G.M. sUNDKLER, Bantu Prophets in South
} Africa

Cambridge, James Clarke \& Co., 2004, 344 p.

Julien Bonhomme

\section{CpenEdition}

Journals

Édition électronique

URL : http://journals.openedition.org/assr/21679

DOI : $10.4000 /$ assr.21679

ISSN : 1777-5825

Éditeur

Éditions de l'EHESS

Édition imprimée

Date de publication : 31 décembre 2009

Pagination : $75-342$

ISBN : 978-2-7132-2218-4

ISSN : 0335-5985

Référence électronique

Julien Bonhomme, «Bengt G.M. sundkLer, Bantu Prophets in South Africa », Archives de sciences sociales des religions [En ligne], 148 | octobre-décembre 2009, document 148-125, mis en ligne le 27 janvier 2010, consulté le 21 septembre 2020. URL : http://journals.openedition.org/assr/21679 ; DOI : https://doi.org/10.4000/assr.21679

Ce document a été généré automatiquement le 21 septembre 2020.

(C) Archives de sciences sociales des religions 


\title{
Bengt G.M. sUNDKLER, Bantu Prophets in South Africa
}

Cambridge, James Clarke \& Co., 2004, 344 p.

\author{
Julien Bonhomme
}

\section{RÉFÉRENCE}

Bengt G.M. SUNDKLER, Bantu Prophets in South Africa, Cambridge, James Clarke \& Co., $2004,344 \mathrm{p}$.

1 En 1948, l'ouvrage de Bengt Sundkler, Bantu Prophets in South Africa, était publié pour la première fois. Force est de constater que, soixante après, l'œuvre reste pleinement actuelle. Son auteur, Suédois né en 1909 et décédé en 1995, est un missionnaire luthérien qui a notamment travaillé en Afrique du Sud, puis en Tanzanie où il a été évêque de Bukoba, avant de devenir professeur d'histoire religieuse à Uppsala. Outre Bantu Prophets in South Africa, assurément son œuvre la plus connue, il est également l'auteur de Zulu Zion and some Swazi Zionists (Oxford University Press, 1976), ainsi que d'un imposant manuscrit sur l'histoire de l'Église en Afrique, publié de manière posthume grâce à l'un de ses étudiants, Christopher Steed: A History of the Church in Africa (Cambridge University Press, 2000). Sundkler peut être considéré à juste titre comme le pionnier de l'anthropologie des «Églises indépendantes africaines», dont Bantu Prophets in South Africa constitue sans conteste la première étude d'envergure. L'ouvrage est d'ailleurs devenu un classique rapidement après sa première publication. Max Gluckman lui-même en fait un compte rendu élogieux dans la revue African Affairs, en 1949, en soulignant sa nouveauté et son intérêt. La postérité du livre est indiscutable: il a fait l'objet de plusieurs rééditions et est abondamment cité aussi bien par les africanistes que, plus largement, par les anthropologues du fait religieux. Sa fameuse distinction entre Églises «sionistes» et «éthiopiennes» est en effet discutée dans la plupart des essais de typologie des nouveaux mouvements religieux africains: voir, par exemple, les articles de James Fernandez, «African Religious Movements. 
Types and Dynamics» (Journal of Modern African Studies, II-4, 1964, pp.531-549) et Vittorio Lanternari, «Syncrétismes, messianismes, néo-traditionalismes en Afrique Noire» (Archives de Sciences Sociales des Religions, 19, 1965, pp.99-116). En France, c'est Georges Balandier qui, le premier, a fait connaître les travaux de Sundkler qu'il cite, dès 1951, dans son célèbre article sur la «situation coloniale» paru dans les cahiers internationaux de sociologie (même si le nom de l'auteur y est orthographié de manière fautive Stukler), puis à nouveau dans «Messianismes et nationalismes en Afrique noire» (1953) et Sociologie actuelle de l'Afrique noire (1955). Il reconnaît dans Bantu Prophets in South Africa «une analyse sociologique de grande portée» qui contribue intelligemment à replacer l'émergence de nouveaux mouvements religieux dans le cadre des réactions africaines à la domination coloniale. À cet égard, on peut d'ailleurs regretter que l'éditeur de cette nouvelle republication n'ait pas jugé bon d'inclure une préface qui retrace la postérité scientifique de l'ouvrage et justifie l'intérêt de sa réédition soixante ans après. On déplorera, en outre, qu'il nous propose un fac-similé de l'édition originale de 1948 et non de l'édition révisée et augmentée de 1961. Cette seconde édition incluait, en effet, un nouveau chapitre conclusif d'une quarantaine de pages portant sur la période 1948-1960. C'est là assurément la principale lacune de cette republication.

2 L'ouvrage est tout entier consacré aux Églises indépendantes en Afrique du Sud, et plus précisément en pays zulu. L'analyse repose sur les observations recueillies par l'auteur au cours des dix années qu'il a passées comme missionnaire parmi les Zulu, mais aussi sur celles collectées par ses dix assistants de terrain, eux-mêmes zulu. Le grand intérêt du travail de Sundkler est, alors, d'articuler conjointement vue d'ensemble et vue de détail. L'étude embrasse, en effet, près de huit cents Églises différentes (dont la liste est donnée en annexe) dans une perspective à la fois sociologique et historique; mais elle offre également des données ethnographiques plus détaillées sur certaines congrégations, par exemple sur la communauté amaNazaretha fondée par le prophète Isaiah Shembe (1870-1935). L'auteur insiste particulièrement sur le «séparatisme» de ces Églises africaines: elles sont en effet nées, à la fin du XIX siècle, d'une sécession avec les missions européennes implantées en Afrique du Sud. S'appuyant sur le congrégationalisme cher aux protestants, les Africains revendiquent le droit à former eux-mêmes des congrégations autonomes. Cette revendication est d'ailleurs défendue par certains missionnaires, aussi bien au nom de l'indépendance des Africains que de la ségrégation, puisqu'il s'agit de permettre désormais aux populations locales de fréquenter des Églises «parallèles» à celles fréquentées par les Européens. Sundkler replace ainsi ce séparatisme religieux dans le cadre des relations entre Européens et Africains au sein de la société sud-africaine - l'auteur ne cachant pas son hostilité personnelle à l'égard de la politique d'apartheid (qui est officiellement mise en place l'année même de la publication de l'ouvrage). Les Églises indépendantes sont en effet particulièrement vivaces en Afrique du Sud en raison de la discrimination raciale (colour bar) qui y sévit, y compris à l'intérieur des paroisses. Ce n'est alors pas un hasard si, au moment même où sont appliquées les premières mesures discriminatoires après la création de l'Union Sud-Africaine, apparaissent des mouvements prophétiques qui annoncent la subversion de l'ordre social et l'expulsion imminente des Européens. C'est bien là ce qui fait l'évidente dimension politique des Églises indépendantes africaines. C'est d'ailleurs pour cette raison que les autorités ont tenté de réprimer ces mouvements religieux, y compris par la violence, avant de chercher plutôt à les encadrer et les contrôler. En 1918, soixante-seize Églises indépendantes sont recensées par les autorités; en 1945, elles sont désormais près de huit cents. Toutefois, seules huit 
d'entre elles sont officiellement reconnues, ce qui montre le caractère somme toute très limité de cette politique d'encadrement. La reconnaissance officielle entraîne pourtant de nombreux privilèges: l'accès à des terrains, le droit de célébrer les mariages, mais aussi l'autorisation d'acheter du vin de messe (le Liquor Act interdisant normalement la vente d'alcool européen aux Africains).

3 L'étude de Sundkler ne se limite toutefois pas aux seuls rapports entre les Églises indépendantes et les autorités sud-africaines. L'auteur a en effet le souci constant de replacer les Églises dans leur contexte socioculturel local. Il s'intéresse ainsi aux relations variables entre l'Église et la communauté environnante dans laquelle elle se situe. Dans la réserve zulu du Natal, il y a, par exemple, de fréquentes tensions entre les leaders religieux et les chefs tribaux, notamment au sujet du statut des femmes qui occupent une place importante dans les congrégations. Dans les exploitations agricoles rurales, la situation sociale n'est pas la même: les tensions concernent davantage les leaders religieux et les missionnaires ou les fermiers blancs. Mais Sundkler s'intéresse également à l'organisation sociale des congrégations et à la sociologie des fidèles: il fournit des données précises concernant le budget dont disposent les congrégations, la composition de leur clergé, leur implantation géographique, le nombre des fidèles, leur sex ratio, leur niveau d'éducation, etc. Cette quantification sociologique n'exclut d'ailleurs pas l'humour, comme lorsque l'auteur remarque à propos des effectifs improbables revendiqués par une communauté: «il serait sans doute plus juste de dire que ces 5000 fidèles habitent en Utopia plutôt qu'en Ouganda» (p.131). C'est toutefois sur la dynamique interne des congrégations que l'étude de Sundkler est sans doute la plus riche et la plus intéressante: il analyse de manière très fine la question de l'autorité du prophète sur les fidèles, ainsi que les crises fissionnelles qui affectent constam-ment les communautés religieuses. Ces dernières connaissent en effet un processus cyclique d'intégration et de désintégration - les principaux motifs de fission étant liés à des problèmes financiers ou sexuels (la question de la polygamie étant un point de litige récurrent). Sundkler montre que ce processus social emprunte à la fois au modèle de la fission segmentaire des Kraal zulu et à celui du schisme congrégationnel protestant. Le schisme constitue en quelque sorte une norme attendue, ce qui explique la prolifération de nouvelles congrégations. Cette multiplication des Églises produit une situation de concurrence intense et favorise par conséquent les itinéraires de vagabondage spirituel (spiritual vagrancy) des fidèles d'une communauté à l'autre. L'appartenance à une Église est donc souvent flottante. Certaines communautés religieuses se retrouvent même parfois privées de meneur (leaderless groups) et se mettent alors en quête d'un nouveau prophète à suivre.

4 C'est assurément pour sa typologie des Églises indépendantes africaines que le travail de Sundkler est le plus connu. Parmi la pléthore des congrégations zulu, il distingue deux types distincts: les Églises de type «éthiopien» et les Églises de type «sioniste». Les termes choisis sont cependant quelque peu trompeurs, puisque les Églises éthiopiennes n'ont pas directement à voir avec l'Éthiopie et que les Églises sionistes n'ont strictement rien à voir avec le sionisme juif. Ces épithètes circonstancielles proviennent en réalité du nom des congrégations à l'origine des différentes Églises des deux types. L'Ethiopian Church naît, en 1892, au Witwatersrand, sous l'influence de missionnaires wesleyens. Le terme «éthiopien» est une mention biblique du Livre des Psaumes et désigne de manière métonymique l'Afrique en général plutôt qu'un pays particulier (même si à partir de la résistance victorieuse des Éthiopiens à la colonisation et de la guerre italo-abyssinienne, le pays acquiert une aura toute 
singulière aux yeux des Africains). L'Ethiopian Church entre rapidement en relation avec l'Église méthodiste épiscopale africaine, fondée aux États-Unis en 1816 par des Noirs et pour les Noirs. Dans les décennies suivantes, elle donne naissance à une série de nouvelles Églises indépendantes désormais coupées des congrégations missionnaires (Sundkler en dresse l'arbre généalogique à la page 44). Ces Églises éthiopiennes qui entretiennent l'idéal d'une «nation chrétienne africaine» se caractérisent par un séparatisme résolument affirmé (même si elles copient leur organisation et leur activité religieuse sur les missions européennes dont elles se sont détachées). En l'absence de toute autre opportunité politique du fait de la discrimination raciale, ces Églises offrent ainsi un cadre possible d'expression à des revendications nationalistes.

Les Églises de type sioniste proviennent, quant à elles, de la Christian Catholic Apostolic Church in Zion, fondée en 1896 aux États-Unis (on mesure ainsi l'importance déterminante des Églises noires américaines pour comprendre la situation des Églises indépendantes africaines). Le terme "sioniste» est là encore une référence biblique qui renvoie à Jérusalem et à la Terre promise. La Christian Catholic Apostolic Church in Zion s'implante en Afrique du Sud à partir de 1904 et donne naissance à une myriade de nouvelles Églises indépendantes, tout en intégrant une forte influence pentecôtiste. Ces Églises sionistes se caractérisent par l'importance centrale qu'y joue la guérison divine (un prophète déclare même: «ceci n'est pas une église, c'est un hôpital»), ainsi que par leur tonalité messianique (les fidèles entretiennent la croyance dans le retour imminent du Christ et sont obsédés par la Nouvelle Jérusalem). Le messianisme sioniste véhicule lui aussi une idéologie à valeur politique, notamment à travers la figure d'un Messie spécifiquement africain. Les prophètes vont jusqu'à dénoncer Jésus qui ne serait qu'un faux prophète pour les Blancs et ne concernerait pas les Noirs. Une fidèle affirme, par exemple, à propos du prophète Isaiah Shembe: «Jésus, nous ne l'avons jamais vu qu'en photo! Alors que je connais Shembe et je crois en lui. C'est lui qui a créé le ciel et la terre; il est Dieu pour nous les Noirs» (cité p.281). De même, plusieurs prophètes sionistes déclarent sans ambages: «Je suis Jésus», se plaçant en rivalité directe avec le Christ des missions. Les représentations du Paradis au sein des Églises sionistes illustrent bien cet antagonisme. Les douze portes du Paradis sont réduites à deux portes, une pour chaque «race»: le prophète Shembe affirme, par exemple, qu'«une race ne peut pas entrer par la porte de l'autre race» (cité p.291). Et parfois, le Paradis ne possède plus même qu'une seule porte qui est alors réservée aux Noirs: l'imaginaire du Paradis offre ainsi l'espoir d'une inversion de la barrière raciale (colour bar).

6 Pour fonder sa typologie, Sundkler s'attache à repérer un certain nombre de contrastes significatifs entre les Églises de type sioniste et celles de type éthiopien. Ainsi, les femmes sont bien davantage présentes dans les premières que dans les secondes. Elles y occupent parfois même la place de leader de la communauté. C'est d'ailleurs là un des registres essentiels de la transformation du statut de la femme dans la société zulu. La féminisation des congrégations sionistes s'accompagne d'un style religieux particulier qui accorde une large place à la transe, aux glossolalies et à la possession par l'esprit saint. Le style du leadership diffère également entre les Églises sionistes et éthiopiennes, les premières étant plus «populaires» et les secondes plus «aristocratiques». D’une manière générale, l'exercice de l'autorité au sein des Églises est écartelé entre l'idéal démocratique des congrégations protestantes et le système hiérarchique de la royauté zulu. Au sein des Églises éthiopiennes, le leader exerce sa domination sur le modèle du chef tribal zulu, dans une relation à la fois mimétique et 
hostile à l'égard du pouvoir des Blancs. Le leader éthiopien a tendance à rester dans son bureau, à l'écart des fidèles, pour administrer l'Église sur un mode qui se veut moderne et rationnel. À l'inverse, le leader de type sioniste entretient des contacts très directs avec ses fidèles, notamment à travers les pratiques de guérison. Le leader sioniste est ainsi un véritable prophète charismatique, qui n'est d'ailleurs pas sans ressemblance avec le devin-guérisseur traditionnel (isangoma). Entre ces deux types-idéaux de leaders religieux, Sundkler note qu'il existe toute une gamme de figures hybrides qui empruntent à la fois aux deux modes d'autorité. Il montre comment un leader est parfois amené à transformer le style de son leadership dans le but de maintenir son autorité sur la communauté des fidèles.

7 L'analyse typologique de Sundkler passe également par une description très précise du culte au sein des Églises indépendantes. Contrairement à l'anti-ritualisme des missions protestantes, les Églises indépendantes se révèlent ultra-ritualistes et insistent fortement sur l'orthopraxie liturgique. De ce point de vue, les Églises sionistes sont sans doute plus riches que celles de type éthiopien - leur liturgie spécifique ayant d'ailleurs tendance à s'imposer progressivement à toutes les Églises. Le style religieux des congrégations sionistes se caractérise par son effervescence émotionnelle: les pleurs, les transes, les glossolalies ou les exorcismes - parfois spectaculaires et violents - y occupent une place déterminante. Les communautés sionistes ont remplacé le sermon des Églises éthiopiennes par la confession, livrée sur un mode assez stéréotypé mi-parlé mi-chanté, par des fidèles qui viennent témoigner publiquement de leur guérison divine. Dans sa description des pratiques rituelles, Sundkler insiste tout particulièrement sur un sous-type des Églises sionistes: les communautés dites «Bethesda». Il s'agit de congrégations entièrement centrées autour de la guérison miraculeuse, notamment par le biais de rites de purification par immersion ou de l'imposition des mains. Dans l'Évangile de Jean, la "piscine de Bethesda» désigne, en effet, le «lieu de la grâce» où le Christ a guéri un paralytique. Les prophètes-guérisseurs des communautés Bethesda se reconnaissent ainsi dans la figure de Jean le Baptiste et comparent explicitement au Jourdain les rivières dans lesquelles ils pratiquent leurs rites baptismaux. La plupart des fidèles viennent donc dans ces Églises, poussés par la maladie ou l'infortune (Sundkler détaille d'ailleurs plusieurs itinéraires thérapeutiques individuels). Ces malades sont majoritairement des femmes et leurs maux touchent avant tout des problèmes de stérilité. La conception de la maladie, du malheur et du mal au sein des communautés Bethesda tourne autour de la notion de souillure: un malade est avant tout quelqu'un qui «a le corps sale». L'immersion, dans l'eau vive notamment, permet alors de laver le corps et, partant, de chasser la souillure. Les émétiques et les purgatifs jouent également un rôle purificateur important dans les communautés Bethesda, et une congrégation a même choisi pour nom: «The African Castor Oil Dead Church» (castor oil désignant l'huile de ricin)! De même, le savon est couramment utilisé dans les rites de purification, qui associent généralement pureté et blancheur (le blanc étant la couleur privilégiée des robes des adeptes, alors que le noir et le rouge sont proscrits). Tous ces rites de purification s'accompagnent de pratiques de confession. Les communautés Bethesda imposent enfin à leurs fidèles de nombreux interdits (alimentaires et sexuels notamment).

$8 \quad$ Les Églises de type Bethesda forment de véritables colonies dans lesquelles les malades vivent à demeure pendant des mois, voire des années. Ces Églises de guérison ressemblent en cela aux communautés thérapeutiques des devins-guérisseurs traditionnels, alors même que leurs prophètes affirment avec force qu'ils n'ont rien à 
voir avec elles. Certes, les médecines traditionnelles (muthi), de même que les cultes thérapeutiques de possession, sont jugés «sataniques»; mais les médicaments européens font également l'objet d'un rejet. Le prophète sioniste condamne ouvertement les rites "païens», mais calque en réalité son activité sur celle du devinguérisseur: il utilise les mêmes armes que lui et s'avère tout autant obsédés par la sorcellerie. Pour qualifier cette proximité ambivalente entre le prophète-guérisseur et le devin contre-sorcier, Sundkler propose de reprendre la fameuse formule: «Plus ça change, plus c'est la même chose». Il ouvre ainsi une réflexion sur la question du syncrétisme (spécifiquement abordée dans le dernier chapitre intitulé: «Du vin nouveau dans de vieilles outres»). Les Églises sionistes associent en effet des éléments chrétiens (pentecôtistes notamment) et des éléments zulu pour donner naissance à une forme rituelle inédite. Sundkler dégage ainsi tout un jeu de correspondances entre le système religieux traditionnel et le système chrétien: les ancêtres sont associés à l'esprit saint, les esprits aquatiques deviennent des anges. Mais il montre également que le syncrétisme des Églises indépendantes peut être envisagé comme une sorte de retour ambivalent au paganisme par rapport aux missions - et c'est là ce qui fait la dimension «nativiste» des Églises sionistes.

9 En conclusion, Bantu Prophets in South Africa constitue une magnifique étude des Églises indépendantes africaines, qui se distingue par l'ampleur et la précision des données ethnographiques et de leur analyse. Il est d'ailleurs notable que nombre de thèmes abordés par Sundkler ont été depuis lors largement repris et fouillés dans les travaux en sciences sociales des religions: par exemple, les Églises de guérison, le syncrétisme, l'importance de la sorcellerie dans les Églises africaines ou encore la dimension politique des prophétismes. L'approche théorique adoptée par l'auteur conserve une étonnante actualité pour un texte écrit il y a plus de soixante ans. L'analyse des dynamiques sociales des communautés religieuses fait penser au meilleur Max Gluckman - d'ailleurs cité à de nombreuses reprises. Mais l'ouvrage possède également une tonalité très wébérienne - alors même que Max Weber n'est pas une fois cité: ainsi la démarche idéal-typique (accompagnée d'une réflexion sur les types intermédiaires et les passages d'un type à un autre), mais aussi l'analyse des différents styles d'autorité ou encore de la figure du prophète charismatique. L'une des questions posées par l'ouvrage, mais que l'auteur n'aborde pas directement, concerne évidemment le degré de généralité de la typologie «sioniste»/ «éthiopien». Certes, les tentatives de généralisation ne sont pas facilitées par le choix de termes circonstanciels peu explicites pour nommer les deux idéaux-types (plutôt que de vocables renvoyant aux propriétés saillantes respectives des types). On ne saurait transposer sans précaution cette typologie à d'autres époques ou d'autres aires culturelles (même africaines), dans la mesure où les mouvements religieux étudiés par Sundkler ne peuvent être compris hors de la société zulu et de son enchâssement dans la société sud-africaine des $\mathrm{xIX}^{\mathrm{e}}$ et $\mathrm{xx}^{\mathrm{e}}$ siècles. Cela dit, en mettant l'accent sur certaines variables socioculturelles pertinentes (type de leadership, place de la guérison, dimension syncrétique, rapport à la tradition, etc.), Sundkler offre clairement des pistes de généralisation possible. 